\title{
Feasibility study of hospital antimicrobial stewardship analytics using electronic health records
}

\author{
P. F. Dutey-Magni $\mathbb{D}^{1 *}$, M. J. Gill ${ }^{2}$, D. McNulty ${ }^{2}$, G. Sohal ${ }^{2}$, A. Hayward ${ }^{3}$ and L. Shallcross $\mathbb{D}^{1}$ on behalf of the PASS \\ research groupt
}

\begin{abstract}
${ }^{1}$ Institute of Health Informatics, University College London, London, UK; ${ }^{2}$ University Hospitals Birmingham NHS Foundation Trust, Birmingham, UK; ${ }^{3}$ Institute of Epidemiology \& Health Care, University College London, London, UK
\end{abstract}

*Corresponding author. E-mail: p.dutey-magni@ucl.ac.uk

†Members are listed in the Acknowledgements section.

Received 6 October 2020; accepted 27 January 2021

\begin{abstract}
Background: Hospital antimicrobial stewardship (AMS) programmes are multidisciplinary initiatives to optimize antimicrobial use. Most hospitals depend on time-consuming manual audits to monitor clinicians' prescribing. But much of the information needed could be sourced from electronic health records (EHRs).
\end{abstract}

Objectives: To develop an informatics methodology to analyse characteristics of hospital AMS practice using routine electronic prescribing and laboratory records.

\begin{abstract}
Methods: Feasibility study using electronic prescribing, laboratory and clinical coding records from adult patients admitted to six specialities at Queen Elizabeth Hospital, Birmingham, UK (September 2017-August 2018). The study involved: (i) a review of AMS standards of care; (ii) their translation into concepts measurable from commonly available EHRs; and (iii) a pilot application in an EHR cohort study ( $n=61679$ admissions).

Results: We developed data modelling methods to characterize antimicrobial use (antimicrobial therapy episode linkage methods, therapy table, therapy changes). Prescriptions were linked into antimicrobial therapy episodes (mean 2.4 prescriptions/episode; mean length of therapy 5.8 days), enabling several actionable findings. For example, $22 \%$ of therapy episodes for low-severity community-acquired pneumonia were congruent with prescribing guidelines, with a tendency to use broader-spectrum antibiotics. Analysis of therapy changes revealed IV to oral therapy switching was delayed by an average 3.6 days (95\% CI: 3.4-3.7). Microbial cultures were performed prior to treatment initiation in just $22 \%$ of antibacterial prescriptions. The proposed methods enabled fine-grained monitoring of AMS practice down to specialities, wards and individual clinical teams by case mix, enabling more meaningful peer comparison.
\end{abstract}

Conclusions: It is feasible to use hospital EHRs to construct rapid, meaningful measures of prescribing quality with potential to support quality improvement interventions (audit/feedback to prescribers), engagement with front-line clinicians on optimizing prescribing, and AMS impact evaluation studies.

\section{Introduction}

The aims of antimicrobial stewardship (AMS) are 'first, to ensure effective treatment of patients with infection, and second, to minimize collateral damage from antimicrobial use. ${ }^{1}$ Hospital AMS guidelines ${ }^{2-7}$ recommend regular clinical audits of prescriptions and feedback of results to prescribers by infection specialists. Yet, doing so is labour-intensive and dependent on specialist expertise, ${ }^{8}$ as it involves reviewing what diagnostic tests were performed and assessing the compliance with local prescribing guidelines. Similarly, point prevalence surveys conducted for infection surveillance ${ }^{9}$ can be prohibitive both in terms of professional time and methodological skill. This hinders hospitals' capacity to monitor prescribing on a large scale. ${ }^{8,10,11}$

Electronic health records (EHRs) collected routinely by hospital information systems offer potential solutions to this problem. King et $a l^{12}$ and Hand et al. ${ }^{13}$ scoped the potential role of electronic prescribing software in supporting prescribers across the full antibiotic lifecycle (prescription initiation, review, discontinuation and dispensing of discharge medications). Other studies have demonstrated the feasibility of using computerized laboratory results, including microbial cultures and sensitivities, to guide the choice of antimicrobial agent in empirical therapy ${ }^{14}$ and increase the proportion of cases treated with effective antimicrobials. ${ }^{15}$

(c) The Author(s) 2021. Published by Oxford University Press on behalf of the British Society for Antimicrobial Chemotherapy.

This is an Open Access article distributed under the terms of the Creative Commons Attribution License (http://creativecommons.org/licenses/ by/4.0/), which permits unrestricted reuse, distribution, and reproduction in any medium, provided the original work is properly cited. 
EHRs thus have the potential to enable a range of functions recommended in AMS guidelines, ${ }^{2}$ particularly: audit of practice, feedback to prescribers, and infection surveillance (tracking syndromes, pathogens and susceptibility). Despite this, the use of EHRs to drive AMS programmes remains 'underexploited'. ${ }^{16}$ Extraction of records is challenging, ${ }^{17}$ resulting in very limited secondary use for evidence-based medicine. ${ }^{18}$ In response, the UK's Antimicrobial Resistance National Action Plan set goals for a comprehensive use of EHRs to 'support and drive good antimicrobial stewardship by coding, auditing and providing feedback for surveillance' by 2025 . $^{16}$

The aim of the present paper was to assess the feasibility of auditing antimicrobial stewardship practices using routinely collected EHRs in order to provide relevant information to different AMS stakeholders including clinicians, hospital managers and policymakers. Key objectives were to: (i) infer the indication of antibiotics prescribed to inpatients; (ii) assess the congruence of individual prescriptions with local prescribing guidelines, particularly in relation to empirical therapy; (iii) compute metrics of stewardship beyond consumption of antibiotics; and (iv) compare these metrics between specialities and between consultant teams within specialities.

This feasibility study followed three steps. First, we synthesized concepts relevant to antimicrobial stewardship performance from clinical guidelines and infection surveillance protocols and translated them into operational definitions applicable to EHRs. Second, we modelled and visualized records to refine definitions that could be applied to data from one specialist hospital in Birmingham, UK. Third, we computed AMS metrics and reviewed compliance of clinical practices with AMS guidelines.

\section{Materials and methods}

\section{Ethics}

This research was approved by University College London's Research Ethics Committee (REC reference 16765/002). Informed consent was not sought for the secondary analysis of pseudonymized EHRs.

\section{Study design and population}

We conducted a retrospective cohort study of records corresponding to episodes of care in six specialities (general medicine, respiratory medicine, geriatric medicine, cardiology, general surgery, urology) at Queen Elizabeth Hospital Birmingham (QEHB) for adult inpatients admitted between 1 September 2017 and 31 August 2018 ( $n=61679$ admissions). QEHB is a specialist teaching hospital in Birmingham, UK with over 1000 general and acute inpatient beds.

\section{Variables}

Pseudonymized EHRs consisted of patient demographics, clinical diagnosis codes (ICD-10, ${ }^{19}$ reclassified as shown in Table S3, available as Supplementary data at JAC-AMR Online), clinical procedure codes (OPCS4), ${ }^{20}$ episodes of care (pseudonymized consultant code, consultant speciality), ward movements, and key investigation results (blood counts, vital signs, blood pressure, organ function).

Antibacterial drug prescription and administration records were extracted from QEHB's locally developed Prescribing, Information and Communication System (PICS). ${ }^{21}$ PICS follows the common UK 'dosebased' prescribing approach, ${ }^{22}$ in which prescribers issue a request containing one or more drug names (Trade Family), dose, route and frequency.

Microbial culture results, including no-growth results and cultures ordered by general practitioners were extracted from PICS. We applied
EUCAST interpretative criteria, $^{23}$ and classified bacterial isolates by MDR profile (multiple, extensive and pan-drug resistance) according to rules set out by Magiorakos et al. ${ }^{24}$ CURB- $65,{ }^{25}$ an important risk stratification score for community-acquired pneumonia (CAP), was computed without the confusion score due to lack of reliable data.

\section{AMS metrics}

Relevant definitions and standards of care were identified from international hospital antimicrobial stewardship and infection treatment guidelines using a list systematically compiled in $2018,{ }^{26}$ alongside four UK-specific reference sources. ${ }^{2,9,27,28}$ We narrowed down a list of measures (Table 1) on the basis of (i) their relevance to inform a hospital AMS strategy and (ii) the availability of sufficient information to measure them within commonly encountered EHRs. These measures characterize the following:

- Antimicrobial consumption (dose and duration). Defined daily doses, days of therapy (DOT), and length of therapy (LOT: duration of the episode, irrespective of the number of antimicrobials administered concurrently) were calculated and aggregated by ward, speciality, consultant teams, and clinical indication as per definitions by Ibrahim et al. ${ }^{29}$

- Changes of therapy tracked changes in antibacterial treatment choices across a 'therapy episode' (Data modelling section) and their timing relative to microbiological outcomes and clinical progression. One such change, de-escalation, is recommended when microbial culture and susceptibilities are available, or when there is limited evidence of infection. It is most easily measured in antibiotics with the broadest spectrum of activity, where only a small number of other drugs would have equivalent spectrum. Conversion from IV therapy to oral therapy is another commonly recommended change of therapy intervention, which can facilitate discharge and reduce some adverse effects of injections. ${ }^{30}$ We computed the time by which criteria for switching from IV to oral regimens were met, based on a set of 'ABCD' criteria listed in QEHB's antimicrobial prescribing guidelines (Table 2), some of which are in common with the Glasgow Audit Tool. ${ }^{28}$ Out of these, ability to take oral medication (criterion B) could not be assessed from records, but other criteria could be measured continuously.

- Congruence of practice with prescribing guidelines. Prescriptions starting a therapy episode were compared with first-line choice of empirical therapy recommended in local prescribing guidelines.

- Adherence to microbial sampling guidelines recommending submission of bacterial cultures prior to initiation of empirical treatment. ${ }^{27}$ We computed the proportion of prescriptions with a microbial sample taken in the 3 days leading up to antibacterial therapy initiation.

Underlying concepts are defined and mapped to relevant SNOMED CT concept codes $^{31}$ in Table S1.

\section{Data modelling}

Graph theory principles were used to construct periods of uninterrupted antibiotic therapy (therapy episodes) by linking related prescription records. Rule definitions underpinning this linkage are available along with this paper (Appendix S1, Table S2). This enabled identification of sequences of drug administration making up therapy episodes, particularly transition from one class of antimicrobials to another.

For each antimicrobial therapy episode, a dynamic table could be constructed with an hourly resolution capturing changes in therapy in relation to clinical parameters (Table 3). It is used as the basis for analysing changes of therapy in relation to clinical response to treatment (including tracking the timing of conversion from IV to oral therapy administration).

Data processing software was written in Structured Query Language (SQL), R and tidyverse ${ }^{32-34}$ and served as a prototype for the Ramses package. $^{35,36}$ 
Table 1. Overview of antimicrobial stewardship metrics

\begin{tabular}{|c|c|}
\hline Domain & Measures \\
\hline \multirow[t]{4}{*}{ Antimicrobial consumption } & Proportion of hospital admissions with at least one antimicrobial prescription \\
\hline & Mean DOT (total duration of all prescriptions, including where there is overlap, e.g. combination therapy) \\
\hline & Mean LOT (time elapsed between the first and the last drug administration in an episode) \\
\hline & Rate of DOT and LOT per 1000 admissions $^{29}$ \\
\hline $\begin{array}{l}\text { Change of therapy (stop, } \\
\text { switch, continue) }\end{array}$ & $\begin{array}{l}\text { Proportion of first-line monotherapy or combination therapy leading to a different choice of therapy, continu- } \\
\text { ation, or discontinuation }\end{array}$ \\
\hline \multirow[t]{2}{*}{ IV to oral administration switch } & $\begin{array}{l}\text { Proportion of antimicrobial therapy episodes initiated by IV route being subsequently converted in full to oral } \\
\text { route }\end{array}$ \\
\hline & Mean time elapsed between IV therapy initiation and its complete conversion to oral therapy \\
\hline Congruence with guidelines & $\begin{array}{l}\text { Proportion of antimicrobial therapy episodes initiated with one of the first-line treatment options listed in the } \\
\text { local empirical prescribing guidelines }\end{array}$ \\
\hline Microbial culture taking & $\begin{array}{l}\text { Proportion of prescriptions belonging to a therapy episode initiated within } 3 \mathrm{~h} \text { of a blood, urine, skin or sterile site } \\
\text { microbial sample being taken }\end{array}$ \\
\hline
\end{tabular}

Table 2. ABCD criteria: considerations for IV to oral switch (see detailed criteria in Appendix S3)

\begin{tabular}{|c|c|c|}
\hline & Criteria & Markers \\
\hline A & Afebrile for at least $24 \mathrm{~h}$ & Temperature $36^{\circ} \mathrm{C}-38^{\circ} \mathrm{C}$ for $48 \mathrm{~h}$ \\
\hline B & Able to take oral medication (not measured) & $\begin{array}{l}\text { Functional gastrointestinal tract } \\
\text { No malabsorption } \\
\text { No interaction with other medications } \\
\text { Enteral drug form available } \\
\text { Patient can swallow and tolerate oral fluids via a tube }\end{array}$ \\
\hline C & Clinically improving & $\begin{array}{l}\text { No unexplained tachycardia (heart rate less than } 90 \text { beats/min in the } \\
\text { past } 12 \mathrm{~h} \text { ) } \\
\text { Blood pressure stable in the past } 24 \mathrm{~h} \\
\text { Respiratory rate less than } 20 \text { breaths/min in the past } 24 \mathrm{~h} \\
\text { White cell count } 4-12 \times 10^{9} \text { cells/L OR a high white cell count that is falling } \\
\text { Falling C-reactive protein }\end{array}$ \\
\hline D & $\begin{array}{l}\text { Not suffering from certain deep- } \\
\text { seated/high-risk infections }\end{array}$ & $\begin{array}{l}\text { Liver abscess } \\
\text { Osteomyelitis, septic arthritis } \\
\text { Inadequately drained abscesses or empyema } \\
\text { Cavitating pneumonia } \\
\text { Staphylococcus aureus bacteraemia } \\
\text { Severe necrotizing soft tissue infections } \\
\text { Severe infections during chemotherapy related neutropenia } \\
\text { Infected implants/prosthesis } \\
\text { Meningitis/encephalitis } \\
\text { Intracranial abscesses } \\
\text { Mediastinitis } \\
\text { Endocarditis } \\
\text { Exacerbation of cystic fibrosis/bronchiectasis }\end{array}$ \\
\hline
\end{tabular}

\section{Prescribing indication inference (supervised classification)}

PICS captures drug prescription indications as free text. Such information was not made available to researchers as it contained patient identifiable information. It was also affected by a high prevalence of missing data (in the region of 50\%). In order to demonstrate our approach, drug indications were instead classified retrospectively using a training dataset that had been collected during an audit of antibacterial prescribing conducted by pharmacists between 2012 and 2017. This dataset included 4200 prescriptions issued for 2712 patients in the following specialities: general medicine, respiratory medicine, geriatric medicine and general surgery. Pharmacists classified each prescription into 21 possible indications including 'not specified' and 'other' using data in PICS and paper medical records. A total of 463 prescriptions did not have a valid clinical indication, and 364 could not be 
Table 3. Example structure of a therapy table

\begin{tabular}{|c|c|c|c|c|c|c|c|c|}
\hline Patient & Time & Mode & Last WBC & $\begin{array}{l}\text { WBC trend } \\
72 \mathrm{~h}\end{array}$ & $\begin{array}{l}\text { Peak CRP in } \\
\text { last } 72 \mathrm{~h}\end{array}$ & Last CRP & $\ldots$ & $\begin{array}{c}\mathrm{ABCD} \\
\text { criteria met? }\end{array}$ \\
\hline$x$ & 2018-07-31 18:49:51 & IV & 11.0 & -0.05 & 151 & 100 & $\ldots$ & yes \\
\hline$x$ & 2018-07-31 19:49:51 & IV & 8.2 & -0.02 & 151 & 100 & $\ldots$ & yes \\
\hline$x$ & 2018-07-31 20:49:51 & oral & 8.2 & -0.02 & 151 & 40 & $\ldots$ & yes \\
\hline$\cdots$ & $\cdots$ & $\cdots$ & $\cdots$ & $\cdots$ & $\cdots$ & $\cdots$ & $\cdots$ & $\cdots$ \\
\hline
\end{tabular}

WBC, white blood cell count; CRP, C-reactive protein concentration.

linked to electronic prescription records, restricting the analysis to a total of 3228 prescriptions corresponding to 2901 therapy episodes. Indication categories with fewer than 50 episodes (endocarditis, bronchiectasis, diabetic foot and/or osteomyelitis, surgical prophylaxis) were reclassified as 'other'. These records were used as training data to predict the clinical indication across all antimicrobial prescriptions, using random forest classification with a moderate-to-low balanced accuracy of $59 \%$ overall. Predictive analytics were estimated using repeated 5-fold stratified cross-validation and are reported in Appendix S2 and Table $\$ 4$.

\section{Results}

\section{Antimicrobial consumption descriptive characteristics}

A basic characterization of prescribing requires linking prescriptions into episodes of therapy, to describe their duration in relation to patient demographics or type of infection treated. Between 1 September 2017 and 31 August 2018, there were 61679 adult admissions (46853 distinct patients) across the six specialities. Table 4 presents key metrics characterizing antibacterial use (prevalence, duration, quantity) by age group. The mean length of admission was 4.2 days, and 21757 admissions (35\%) contained at least one antibacterial prescription. A total of 59884 antibacterial prescriptions were issued, corresponding to 24511 antibacterial therapy episodes, 141 of which spanned more than one admission. The mean length of antibacterial therapy episodes (LOT) was 5.8 days, equivalent to a mean 8.7 days of therapy (DOT) per admission. The mean DOT increased with age and was significantly higher (9.9 days) in emergency admissions than in elective admissions ( 4.3 days, Figure 1 ).

\section{Changes of therapy}

Changes of therapy could be analysed from the structure of therapy episodes to identify escalation or de-escalation. For instance, therapy episodes initiated with meropenem $(n=969)$ were most commonly: (i) stopped (33\%) after a mean duration of 3.0 days; (ii) continued (28\%) after a mean duration of 2.0 days; (iii) switched to piperacillin/tazobactam (12\%) after a mean duration of 1.1 days; or (iv) switched to co-amoxiclav (9.1\%) after a mean duration of 1.9 days. Outcomes (i), (iii) and (iv) can be regarded as deescalation in this particular instance.

\section{Switch from IV to oral therapy}

Within 16688 out of the 24510 antibacterial therapy episodes, we identified 17614 sequences consisting of one or more IV prescriptions. Overall, 6404 (36\%) of such the IV sequences were converted into oral therapy, with a median and mean duration of IV treatment of 2.4 days and 3.5 days, respectively. On the contrary, 11210 IV sequences (64\%) continued with injections until end of therapy, with a median duration of 1.3 days and a mean duration of 3.5 days. As shown in Figure 2, variation in the conversion to oral therapy across clinical teams and specialities was evident and can be attributed, at least in part, to case mix. For instance, a likely explanation for cardiology's lower conversion rate $(8 \%)$ is that prolonged IV therapy is recommended for deepseated infections such as endocarditis.

We sought to analyse the timeliness of conversion from IV to oral therapy based on ABCD criteria (Table 2). Out of 6404 IV sequences successfully switched, 2670 (42\%) met A, C and D criteria before oral conversion occurred. Out of 11210 sequences never switched, 2682 (21\%) met A, C and D criteria before end of therapy. Across both sets, the delay between criteria being met and end/ conversion of therapy had a median of 2.1 days, a mean of 3.6 days [95\% CI: 3.4-3.7], and a standard deviation of 5.7 days, suggesting considerable variation. Figure 3 presents team- and speciality-level mean delays, suggesting once again some differences between consultant teams within specialities.

\section{Congruence with prescribing guidelines}

We take the example of CAP. In addition to being the most common indication for therapy initiation, CAP prescribing guidelines revolved around a widely adopted risk stratification score (CURB65) which could be measured from EHRs. Of 4222 therapy episodes initiated for CAP, 4109 (97\%) could be linked with a CURB-65 severity score in the $48 \mathrm{~h}$ before or after antibiotic initiation (assuming a mental confusion score of 0 , as this information was not recorded electronically). At the time of prescribing, QEHB guidelines recommended:

- CURB-65 score 0 or 1: amoxicillin; doxycycline (penicillin allergy).

- CURB-65 score 2: amoxicillin/clarithromycin; benzylpenicillin/ clarithromycin; moxifloxacin (penicillin allergy).

- CURB-65 score 3+: co-amoxiclav/clarithromycin; moxifloxacin (penicillin allergy).

Table 5 reports antibiotics initiated as first-line therapy in 2569 low-severity CAP episodes, that is, episodes with a CURB-65 score of 0 or 1 . Out of 927 patients whose CURB- 65 score can confidently be assumed to be at the most 1 (score of 0 when omitting the missing mental confusion score), just 207 (22\%) therapy episodes 
were initiated with the recommended drug, while 331 (36\%) received therapy recommended for higher CURB-65 scores, demonstrating a preference for broad-spectrum antibiotics in prescribers.

\section{Microbial culture taking}

Across a total of 59696 prescriptions ordered by six selected specialities, $22 \%(n=13210)$ were issued after at least one specimen was sampled from blood, drains, respiratory tract, intravascular devices, CNS, aspirates or other tissue or bone samples. Narrowing the criterion to blood samples only, $18 \%(n=10906)$ of all prescriptions and 38\% (1174/3107) of prescriptions for meropenem (mainly used to treat bloodstream infections), could be linked to such a sample. Figure 4 reports findings broken down by speciality and consultant team. Considerable variation can be observed, which could be further examined in relation to variations in therapy indication and compliance with guidelines across specialities/ team.

\section{Discussion \\ Principal findings}

This single-site study demonstrates a pragmatic approach to computing meaningful measures of AMS from electronic prescription, laboratory and hospital care records to support stewardship teams in rapidly identifying areas of prescribing behaviour where there is scope for improved stewardship. In addition to measuring variation in antibiotic use, we demonstrate the feasibility of using routine data to assess overall compliance with guidelines (using the example of (AP) and show how these datasets can be used to compute a range of prescribing metrics (Table 1) built around international AMS recommendations. This can be used to monitor performance; inform the design of stewardship interventions; evaluate their impact; and engage clinical teams in audit and feedback interventions to optimize their prescribing. ${ }^{16}$

\section{Study strengths and limitations}

This study is novel in attempting to measure clinical constructs that normally require manual audits or point prevalence surveys using routinely collected data. ${ }^{28,37}$ We outlined ways of measuring stewardship performance in clinical practice beyond antimicrobial consumption, the main indicator currently used in stewardship surveillance. ${ }^{38}$ National surveillance systems for prescribing and resistance in secondary care provide high-quality measures of resistance and prescribing for policy-makers, but they do not address the needs of front-line clinicians who require more detailed metrics to identify opportunities to improve their performance. This feasibility study demonstrates the potential for locally developed analytics to address the local needs and stewardship priorities of clinicians using routinely collected EHRs. Future iterations of our approach could be expanded to report on the effective and timely use of surgical prophylaxis (and its congruence with guidelines), timely initiation of antimicrobial therapy and adequate empirical therapy coverage of microbial isolates.

Outside of intensive care research, existing literature contains few examples of EHR research simultaneously analysing electronic prescribing, laboratory and care records. To our knowledge, only 


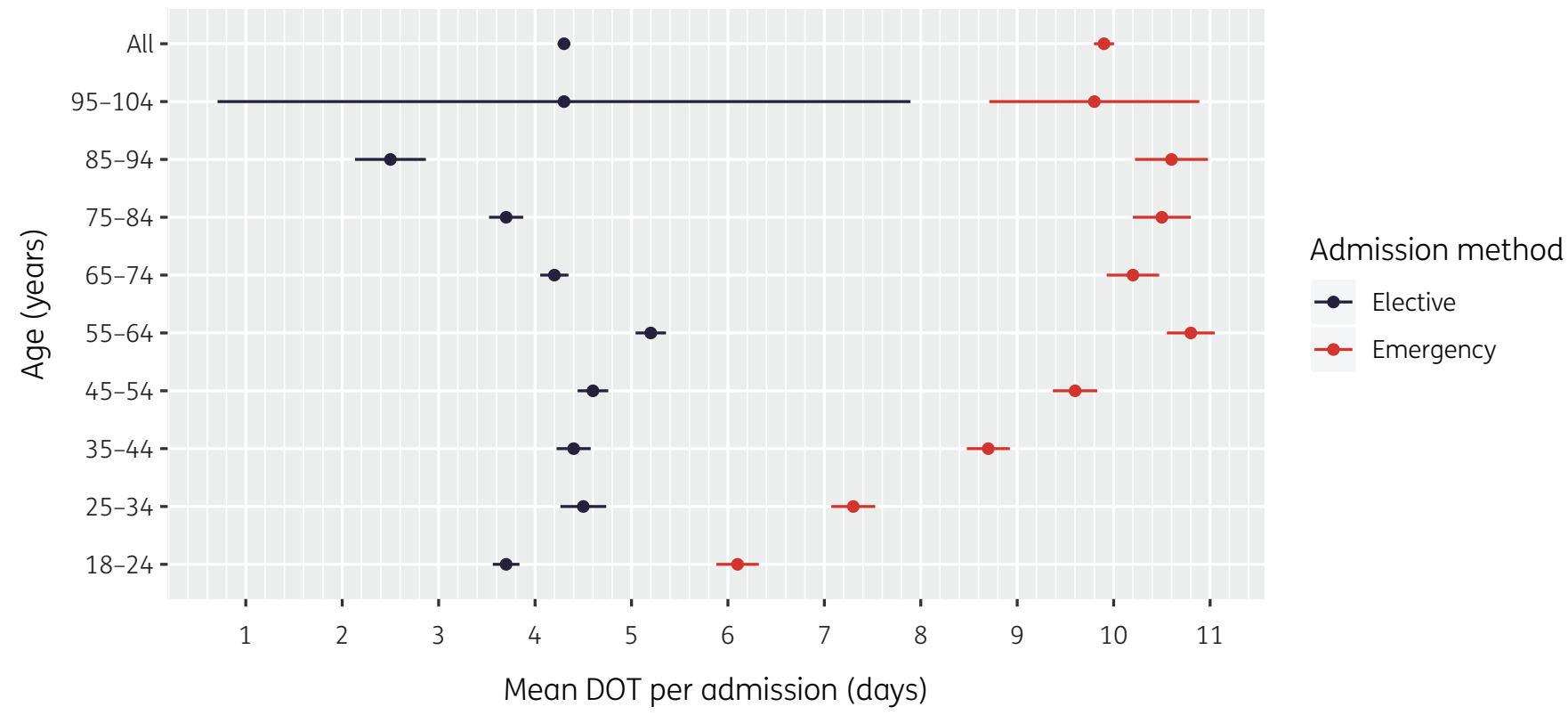

Figure 1. Mean and $95 \%$ CI of the total DOT per admission in patients receiving antimicrobials at any point during an admission (September 2017August 2018).

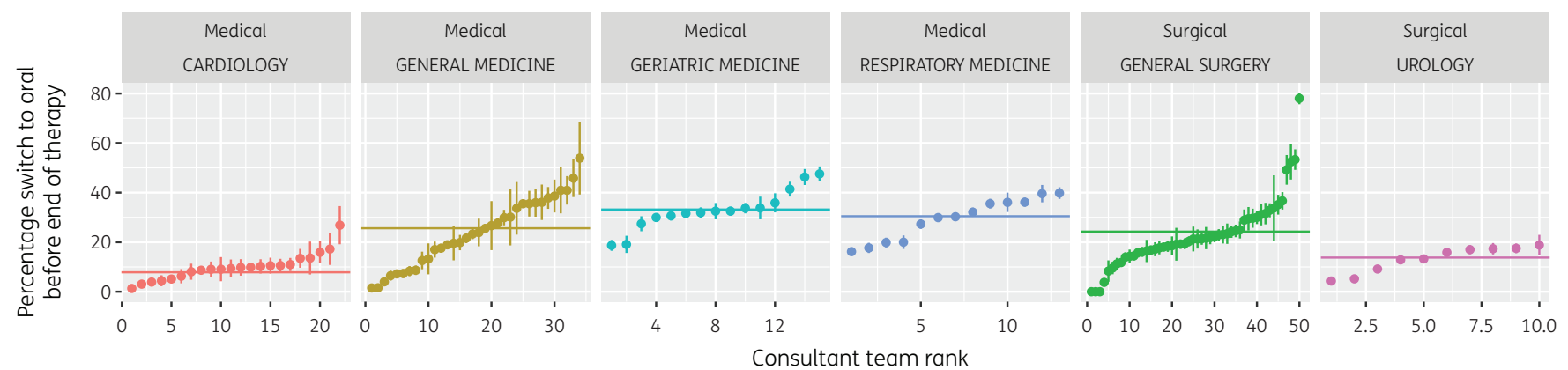

Figure 2. Point and $95 \%$ CI estimates of the proportion of IV therapy converted into oral therapy ranked by consultant team by speciality (September 2017-August 2018). The horizontal line indicates the point estimate for the entire speciality.
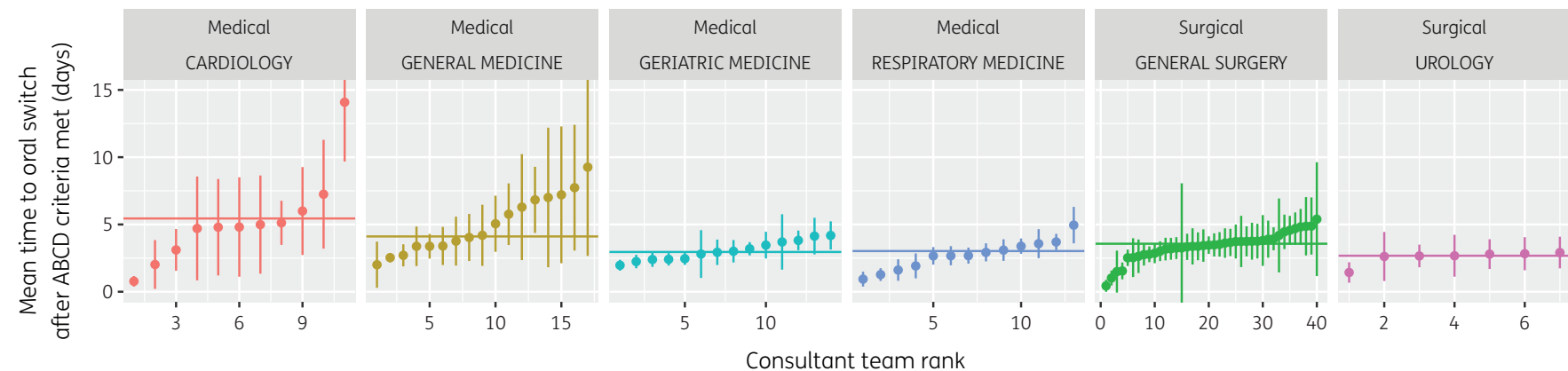

Figure 3. Point and $95 \%$ CI estimates of the mean time (days) elapsed between ABCD criteria being met and conversion to oral therapy, ranked by consultant team by speciality (September 2017-August 2018). The horizontal line indicates the point estimate for the entire speciality.

large bespoke data engineering platforms have achieved this. ${ }^{39-41}$ Unlike the present study, such platforms exploit electronic messages streaming from hospital information systems in real-time: these contain dynamic information, unlike the retrospective view provided from EHRs commonly curated in hospital warehouses. This noteworthy difference has implications: the structure and content of electronic messages tend to be system-specific and require significant investment into developing dedicated data and 
Table 5. First-line therapy choice in CAP episodes in patients with a CURB-65 score of 0 or 1

\begin{tabular}{lcc}
\hline & \multicolumn{2}{c}{$\begin{array}{c}\text { Therapy episodes, } \\
n(\% \text { column total) }\end{array}$} \\
\cline { 2 - 3 } First-line therapy & URB-65 $=0$ & URB-65 = \\
\hline Amoxicillin & $205(22.1)$ & $249(15.5)$ \\
Amoxicillin, clarithromycin & $56(6.0)$ & $104(6.5)$ \\
Azithromycin & $4(0.4)$ & $6(0.4)$ \\
Benzylpenicillin & $2(0.2)$ & $3(0.2)$ \\
Benzylpenicillin, clarithromycin & $14(1.5)$ & $31(1.9)$ \\
Benzylpenicillin, metronidazole & $1(0.1)$ & $0(0.0)$ \\
Ciprofloxacin & $2(0.2)$ & $9(0.6)$ \\
Clarithromycin & $76(8.2)$ & $75(4.7)$ \\
Clarithromycin, co-amoxiclav & $261(28.2)$ & $541(33.8)$ \\
Co-amoxiclav & $104(11.2)$ & $211(13.2)$ \\
Meropenem & $9(1.0)$ & $24(1.5)$ \\
Piperacillin/tazobactam & $2(0.2)$ & $2(0.1)$ \\
Ceftriaxone & $10(1.1)$ & $2(0.1)$ \\
Clarithromycin, moxifloxacin & $2(0.2)$ & $4(0.2)$ \\
Clarithromycin, piperacillin/ & $4(0.4)$ & $9(0.6)$ \\
tazobactam & & \\
Meropenem, vancomycin & $7(0.8)$ & $10(0.6)$ \\
Other & $168(18.1)$ & $322(20.1)$ \\
Total & $927(100)$ & $1602(100)$ \\
& &
\end{tabular}

URB-65, severity score based on CURB- $65,{ }^{25}$ with mental confusion item set to 0: urea (blood urea nitrogen $>7 \mathrm{mmol} / \mathrm{L}$ ) (1 point), respirations per $\min >30$ (1 point), systolic blood pressure $<90 \mathrm{mmHg}$ ( 1 point), age $\geq 65$ years ( 1 point).

analytical models. Such platforms are neither feasible in most hospitals, nor justified for simple surveillance of antibiotic use, stewardship performance and pathogen susceptibility. The pragmatic approach described in the present paper would be accessible to a wider range of hospitals, particularly if interoperable software ${ }^{35,36}$ and code lists/vocabularies are made widely available. In those conditions, a modest proportion of an information analyst's time would be sufficient to validate and map local data to standardized vocabularies and generate comprehensive reports. Metrics specified in Table 1 are designed to be feasible independently of variation in EHRs and vocabularies across hospitals.

This feasibility study reveals the challenges associated with assessing congruence with local prescribing guidelines and the complexity of prescribing decisions. This is partly due to limitations of routine data, but it also reflects a lack of consensus around when and how to de-escalate antibiotics. Manual review of individual prescribing records led authors to conclude that there is too much ambiguity in EHRs to confidently assess the appropriateness of individual prescribing decisions. Prescribing indication data were not available and made it necessary to rely upon statistical classification. This introduced error into the findings: for example, the classifier precision for CAP was 80\% (Table S4), indicating that one in five episodes classified as CAP were likely to have a different indication. However, it is increasingly common for prescribing indication to be recorded in electronic prescribing systems which may make it feasible to assess congruence with prescribing using EHRs alone. Similarly, the lack of access to dispensing records prevented analysis of 'to take away' medications issued at discharge, which can significantly prolong the total LOT. Finally, prescription records were obtained from a snapshot source and did not include a history of changes made to prescriptions' intended duration. This prevented analysis of how frequently prescriptions were stopped early. All analyses were restricted to structured data and did not attempt to derive information that may have been recorded in free text in medical notes.

\section{Implications}

Findings from this feasibility study are now informing the development of an open-source software package ${ }^{35}$ designed to enable hospitals to build their own stewardship analytics using routinely collected EHRs. This has the potential to transform the delivery of stewardship in hospitals by making detailed information on prescribing patterns and resistance widely available in the context of increasing use of electronic prescription, laboratory and hospital care record systems in high-income nations. As of 2020, half of England's acute hospitals had adopted electronic prescribing. ${ }^{42}$ International guidelines ${ }^{2-7}$ recommend local investment into surveillance and analytics to rationalize the use of antimicrobials. In particular, the UK's Antimicrobial Resistance National Action Plan ${ }^{16}$ aims to complete the introduction of electronic prescribing systems across England by 2025, alongside the adoption of international clinical terminology in computerized laboratory systems. ${ }^{43}$ Strong evidence supports the use of feedback to prescribers, ${ }^{2,44}$ but feedback needs to be relevant, targeted (team or individual level), reliable and timely to influence prescribing behaviour. ${ }^{45}$ Further research is needed to statistically adjust those measures for case mix in the same way as consumption measures. ${ }^{46}$ User-centred research ${ }^{47}$ is also needed to tailor these measures to individual clinical teams, AMS teams and hospital managers. There is also a need for research to develop evidencebased standards of care for antimicrobial stewardship, for instance to support decisions around de-escalation. ${ }^{48}$ This could be facilitated by observational studies of routine care records.

\section{Conclusions}

This study shows it is feasible to draw on electronic prescription, laboratory and hospital care records to provide meaningful measures of AMS, by:

(i) Reconstructing 'therapy episodes', which link all relevant prescription records and enable analyses of the length, changes and discontinuation of antimicrobial therapy.

(ii) Inferring the clinical intent and indication of prescriptions (for both monotherapy and combination therapy). We have illustrated the use of supervised classification in general medicine specialities with moderate accuracy for the most common infection categories.

(iii) Computing stewardship performance and quality metrics. Examples include conversion of IV therapy to oral therapy when patients show signs of resolution, microbial culture sampling and congruence with guidelines. 


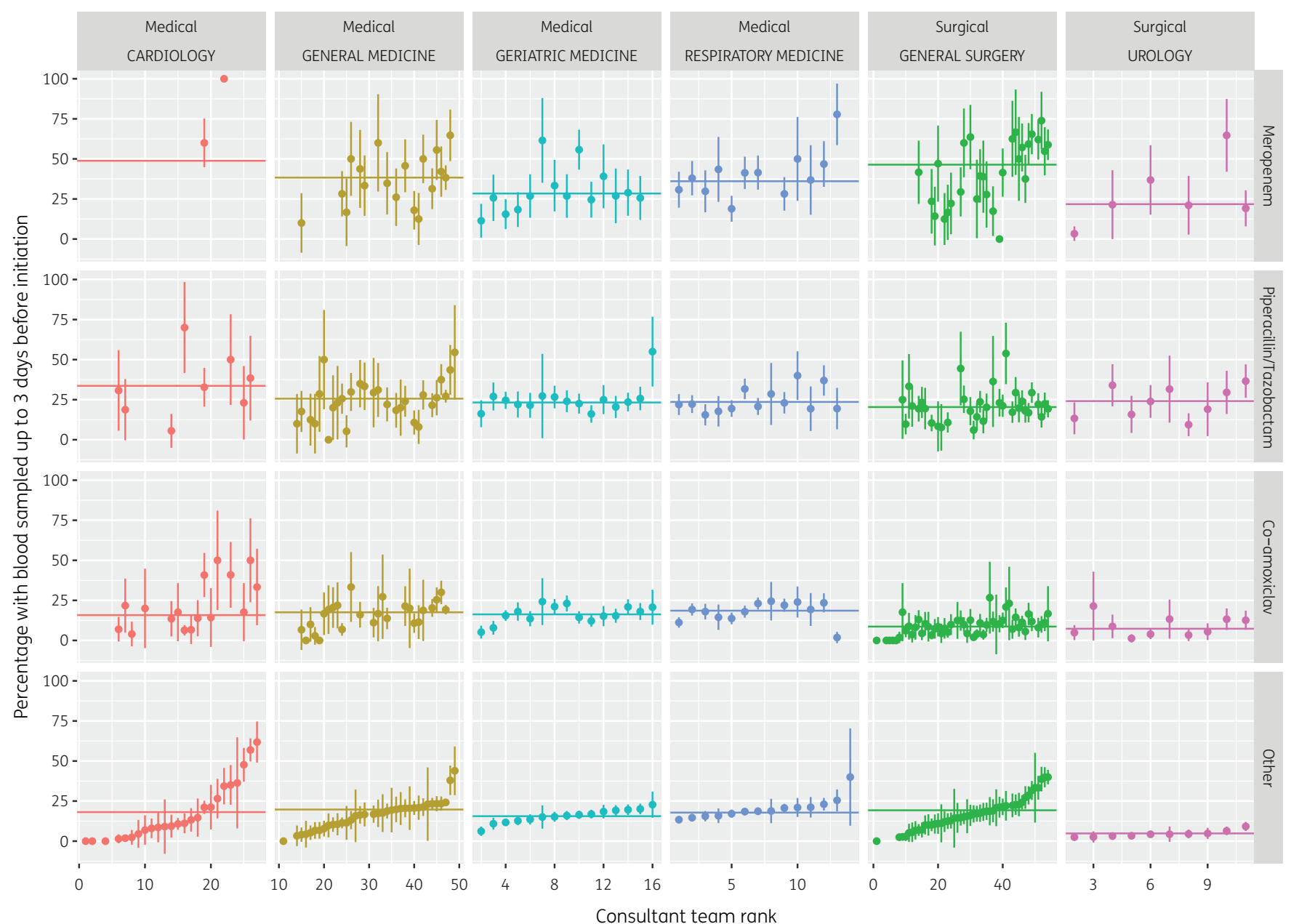

Figure 4. Point and $95 \%$ CI estimates of the proportion of prescriptions initiated with a blood culture sampled in the 3 days leading up to initiation of prescription and/or therapy by consultant team by speciality by drug type in six selected specialities (September 2017-August 2018). Consultant teams are ranked by percentage with a sample for 'other' antibiotic class. The horizontal line indicates the point estimate for the entire speciality.

However, one of the most significant obstacles hindering hospitals' stewardship efforts lies the difficulty in extracting and analysing EHRs from a range of diverse systems. ${ }^{18}$ Reproducible analytical tools are now available to assist microbiology culture and susceptibility analytics. ${ }^{49}$ Software development is underway to support other hospitals in adopting the approach tested in the present study. ${ }^{35,36}$

\section{Acknowledgements}

This work uses pseudonymized data provided by patients and collected by the NHS as part of their care and support. The authors also acknowledge the contribution of staff of the Pharmacy Department at University Hospital Birmingham NHS Foundation Trust, who collected high-quality antimicrobial review outcome data.

\section{Members of the PASS research group}

Niall Anderson, Elise Crayton, Gillian Forbes, Arnoupe Jhass, Emma Richardson, Michelle Richardson, Patrick Rockenschaub, Catherine Smith, Elizabeth Sutton, Rosanna Traina (Investigation); Lou Atkins, Anne Conolly, Spiros Denaxas, Ellen Fragaszy, Rob Horne, Patty Kostkova,
Fabiana Lorencatto, Susan Michie, Jennifer Mindell, John Robson, Claire Royston, Carolyn Tarrant, James Thomas, Jonathan West (Conceptualization, Funding Acquisition and Resources); Haydn Williams (Resources); Nadia Elsay, Chris Fuller (Project Administration).

\section{Funding}

This study was carried out as part of Preserving Antibiotics through Safe Stewardship (PASS), a programme grant funded by the Economic \& Social Research Council (grant reference ES/P008321/1). A.H. is a National Institute for Health Research (NIHR) Senior Investigator. L.S. is funded by a NIHR Clinician Scientist Award (CS-2016-007).

\section{Transparency declarations}

None to declare.

\section{Author contributions}

D.M. extracted data for the study team. M.J.G. extracted and classified microbial culture and susceptibility records. G.S. led the collection of the 
prescription review data. P.F.D.-M programmed and conducted analyses and drafted the manuscript. M.J.G., L.S., A.H. and P.F.D.-M. reviewed visualizations of results and interpreted the findings. All authors read and approved the submitted manuscript.

\section{Disclaimer}

The views expressed in this article are those of the authors and not necessarily those of the NIHR or the Department of Health and Social Care.

\section{Supplementary data}

Tables S1 to S4 and Appendices S1 to S4 are available as Supplementary data at JAC-AMR Online.

\section{References}

1 Davey P, Sneddon J, Nathwani D. Overview of strategies for overcoming the challenge of antimicrobial resistance. Expert Rev Clin Pharmacol 2010; 3: 667-86.

2 NICE. Antimicrobial Stewardship: Systems and Processes for Effective Antimicrobial Medicine Use. NICE guideline NG15. https://www.nice.org.uk/ guidance/ng15.

3 SARI Hospital Antimicrobial Stewardship Working Group. Guidelines for Antimicrobial Stewardship in Hospitals in Ireland. https://www.hpsc.ie/a-z/ microbiologyantimicrobialresistance/infectioncontrolandhai/guidelines/ File,4116,en.pdf.

4 Haute Autorité de Santé. Antibiotic Therapy and Prevention of Bacterial Resistance in Healthcare Organisations. Clinical Practice Guideline 2008. https://www.has-sante.fr/jcms/c_665169/fr/strategie-d-antibiotherapieet-prevention-des-resistances-bacteriennes-en-etablissement-de-sante. 5 Pulcini C, Binda F, Lamkang AS et al. Developing core elements and checklist items for global hospital antimicrobial stewardship programmes: a consensus approach. Clin Microbiol Infect 2019; 25: 20-5.

6 Dellit TH, Owens RC, McGowan JE et al. Infectious Diseases Society of America and the Society for Healthcare Epidemiology of America guidelines for developing an institutional program to enhance antimicrobial stewardship. Clin Infect Dis 2007; 44: 159-77.

7 Stichting Werkgroep Antibiotica Beleid. A Teams Practical Guide. Antimicrobial Stewardship in the Netherlands. https://swab.nl/en/downloadpractice-guide.

8 Chung GWW, Wu JEE, Yeo CLL et al. Antimicrobial stewardship: a review of prospective audit and feedback systems and an objective evaluation of outcomes. Virulence 2013; 4: 151-7.

9 ECDC. Point Prevalence Survey of Healthcare-Associated Infections and Antimicrobial Use in European Acute Care Hospitals - Protocol Version 5.3. https://ecdc.europa.eu/en/publications-data/point-prevalence-survey-health care-associated-infections-and-antimicrobial-use-3.

10 Howard P, Pulcini C, Levy HG et al. An international cross-sectional survey of antimicrobial stewardship programmes in hospitals. J Antimicrob Chemother 2014; 70: 1245-55.

11 Van Gastel E, Costers M, Peetermans WEE et al. Nationwide implementation of antibiotic management teams in Belgian hospitals: a self-reporting survey. J Antimicrob Chemother 2010; 65: 576-80.

12 King A, Cresswell KM, Coleman JJ et al. Investigating the ways in which health information technology can promote antimicrobial stewardship: a conceptual overview. J R Soc Med 2017; 110: 320-9.

13 Hand KS, Cumming D, Hopkins $S$ et al. Electronic prescribing system design priorities for antimicrobial stewardship: a cross-sectional survey of 142 UK infection specialists. J Antimicrob Chemother 2017; 72: 1206-16.

14 Cánovas-Segura B, Campos M, Morales A et al. Development of a clinical decision support system for antibiotic management in a hospital environment. Prog Artif Intell 2016; 5: 181-97.

15 Curtis CE, Al Bahar F, Marriott JF. The effectiveness of computerised decision support on antibiotic use in hospitals: a systematic review. PLoS One 2017; 12: e0183062.

16 UK Department of Health and Social Care. Tackling Antimicrobial Resistance 2019-2024. The UK's Five-Year National Action Plan. https://www. gov.uk/government/publications/uk-5-year-action-plan-for-antimicrobial-re sistance-2019-to-2024.

17 Baysari MT, Lehnbom EC, Li L et al. The effectiveness of information technology to improve antimicrobial prescribing in hospitals: a systematic review and meta-analysis. Int J Med Inform 2016; 92: 15-34.

18 Micallef C, Chaudhry NT, Holmes AH et al. Secondary use of data from hospital electronic prescribing and pharmacy systems to support the quality and safety of antimicrobial use: a systematic review. J Antimicrob Chemother 2017; 72: 1880-5.

19 WHO. The ICD-10 Classification of Mental and Behavioural Disorders. Clinical Descriptions and Diagnostic Guidelines. https://www.who.int/classifica tions/icd/en/bluebook.pdf.

20 Health and Social Care Information Centre. OPCS Classification of Interventions and Procedures Version 4.8 (SCCI0084 Amd 105/2015). https:// digital.nhs.uk/data-and-information/information-standards/informationstandards-and-data-collections-including-extractions/publications-and-noti fications/standards-and-collections/scci0084-opcs-classification-of-interven tions-and-procedures.

21 Nightingale PGG, Adu D, Richards NT et al. Implementation of rules based computerised bedside prescribing and administration: intervention study. BMJ 2000; 320: 750-3.

22 UK Health and Social Care Information Centre. $\mathrm{dm}+\mathrm{d}$ Implementation Guide (Secondary Care) V5.0. https://www.nhsbsa.nhs.uk/sites/default/files/ 2017-02/Secondary_Care_Electronic_Prescribing_Implementation_ Guidance_5_0.pdf.

23 EUCAST. EUCAST Expert Rules Version 3.1. Intrinsic Resistance and Exceptional Phenotypes Tables. http://www.eucast.org/fileadmin/src/ media/PDFs/EUCAST_files/Expert_Rules/Expert_rules_intrinsic_excep tional_V3.1.pdf.

24 Magiorakos A-P, Srinivasan A, Carey RB et al. Multidrug-resistant, extensively drug-resistant and pandrug-resistant bacteria: an international expert proposal for interim standard definitions for acquired resistance. Clin Microbiol Infect 2012; 18: 268-81.

25 Lim WS. Defining community acquired pneumonia severity on presentation to hospital: an international derivation and validation study. Thorax 2003; 58: 377-82.

26 EU-JAMRAI. Work Package 7. List of revised Guidelines; Tools and Implementation Methods for Antibiotic Stewardship: Hospital Care. https:// web.archive.org/web/20201201115136/https://eu-jamrai.eu/wp-content/ uploads/2019/04/EUjamrai_D7.1_RevisedGuidelinesATBS_HOSPITALCare_ WP7_2018.10.31_Rev01.pdf.

27 PHE. Start Smart-Then Focus. Antimicrobial Stewardship Toolkit for English Hospitals, https://www.gov.uk/government/publications/antimicro bial-stewardship-start-smart-then-focus.

28 Seaton RA, Nathwani D, Burton P et al. Point prevalence survey of antibiotic use in Scottish hospitals utilising the Glasgow Antimicrobial Audit Tool (GAAT). Int J Antimicrob Agents 2007; 29: 693-9.

29 Ibrahim OM, Polk RE. Antimicrobial use metrics and benchmarking to improve stewardship outcomes: methodology, opportunities, and challenges. Infect Dis Clin North Am 2014; 28: 195-214. 
30 Athanassa Z, Makris G, Dimopoulos G et al. Early switch to oral treatment in patients with moderate to severe community-acquired pneumonia. Drugs 2008; 68: 2469-81.

31 UK Health and Social Care Information Centre. UK SNOMED CT Drug Extension, RF2: Full, Snapshot \& Delta. https://isd.digital.nhs.uk.

32 R Core Team. R: A Language and Environment for Statistical Computing. https://www.r-project.org/.

33 Wickham H. tidyverse: Easily Install and Load the 'Tidyverse', https:// cran.r-project.org/package =tidyverse.

34 Wickham H, François R, Henry L et al. dplyr: A Grammar of Data Manipulation, https://cran.r-project.org/package=dplyr.

35 Dutey-Magni PF, Shallcross L. Ramses: R Package for Antimicrobial Stewardship \& Surveillance [software]. https://github.com/ramses-antibiot ics/ramses-package/. doi:10.5281/zenodo.4428900.

36 Dutey-Magni PF, Shallcross L. Ramses: R Package for Antimicrobial Stewardship \& Surveillance [software documentation]. https://ramses-antibi otics.web.app/.

37 Charani E, de Barra E, Rawson TM et al. Antibiotic prescribing in general medical and surgical specialties: a prospective cohort study. Antimicrob Resist Infect Control 2019; 8: 151.

38 Vlahović-Palčevski V, Gyssens IC et al. Quality indicators and quantity metrics of antibiotic use. In: Pulcini C, Ergönül Ö, Can F, eds. Antimicrobial Stewardship. Elsevier, 2017; 29-37.

39 Beaudoin M, Kabanza F, Nault V et al. An antimicrobial prescription surveillance system that learns from experience. AI Mag 2014; 35: 15.

40 Lovis C, Colaert D, Stroetmann VNN. DebugIT for patient safety - improving the treatment with antibiotics through multimedia data mining of heterogeneous clinical data. Stud Health Technol Inform 2008; 136: 641-6.

41 Morales A, Cánovas-Segura B, Campos M et al. Proposal of a big data platform for intelligent antibiotic surveillance in a hospital. In: Luaces O, Gámez
JA, Barrenechea E et al. eds. Advances in Artificial Intelligence. CAEPIA 2016. Lecture Notes in Computer Science, Vol 9868. Springer, 2016; 261-270. doi: 10.1007/978-3-319-44636-3 24.

42 Wilkinson E. 'A Blessing and a Curse': The Struggle to Introduce ePrescribing. The Pharmaceutical Journal. https://www.pharmaceutical-jour nal.com/news-and-analysis/features/a-blessing-and-a-curse-the-struggleto-introduce-e-prescribing/20206818.article.

43 NHS Digital. BETA - Clinical Information Standards. https://digital.nhs.uk/ about-nhs-digital/our-work/nhs-digital-data-and-technology-standards/clin ical-information-standards.

44 Ivers N, Jamtvedt G, Flottorp S et al. Audit and feedback: effects on professional practice and healthcare outcomes. Cochrane Database Syst Rev 2012; issue 6: CD000259.

45 Brehaut JC, Colquhoun HL, Eva KW et al. Practice feedback interventions: 15 suggestions for optimizing effectiveness. Ann Intern Med 2016; 164: 435.

46 van Santen KL, Edwards JR, Webb AK et al. The Standardized Antimicrobial Administration Ratio: a new metric for measuring and comparing antibiotic use. Clin Infect Dis 2018; 67: 179-85.

47 Yardley L, Morrison L, Bradbury $\mathrm{K}$ et al. The person-based approach to intervention development: application to digital health-related behavior change interventions. J Med Internet Res 2015; 17: e30.

48 Tabah A, Bassetti M, Kollef MH et al. Antimicrobial de-escalation in critically ill patients: a position statement from a task force of the European Society of Intensive Care Medicine (ESICM) and European Society of Clinical Microbiology and Infectious Diseases (ESCMID) Patients Study Group (ESGCIP). Intensive Care Med 2020; 46: 245-65.

49 Berends MS, Luz CF, Friedrich AW et al. AMR - an R package for working with antimicrobial resistance data. bioRxiv 2019; doi: 10.1101/810622. 